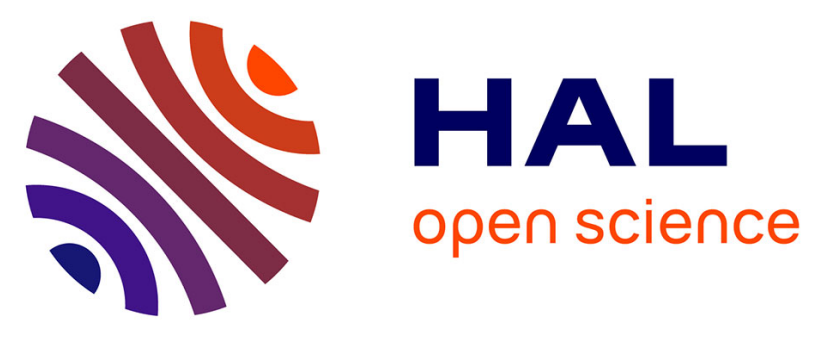

\title{
Risk factors for, and clinical relevance of, faecal extended-spectrum $\beta$-lactamase producing (ESBL-EC) carriage in neutropenic patients with haematological malignancies
}

M. Arnan, C. Gudiol, L. Calatayud, J. Liñares, M. Á. Dominguez, M. Batlle, J. M. Ribera, J. Carratalà, F. Gudiol

\section{To cite this version:}

M. Arnan, C. Gudiol, L. Calatayud, J. Liñares, M. Á. Dominguez, et al.. Risk factors for, and clinical relevance of, faecal extended-spectrum $\beta$-lactamase producing (ESBL-EC) carriage in neutropenic patients with haematological malignancies. European Journal of Clinical Microbiology and Infectious Diseases, 2010, pp.355-360. 10.1007/s10096-010-1093-x . hal-00637076

\section{HAL Id: hal-00637076 https://hal.science/hal-00637076}

Submitted on 30 Oct 2011

HAL is a multi-disciplinary open access archive for the deposit and dissemination of scientific research documents, whether they are published or not. The documents may come from teaching and research institutions in France or abroad, or from public or private research centers.
L'archive ouverte pluridisciplinaire HAL, est destinée au dépôt et à la diffusion de documents scientifiques de niveau recherche, publiés ou non, émanant des établissements d'enseignement et de recherche français ou étrangers, des laboratoires publics ou privés. 
1 Risk Factors for, and Clinical Relevance of, Faecal Extended-Spectrum $\beta$ -

2 Lactamase producing Escherichia coli (ESBL-EC) Carriage in Neutropenic

3 Patients with Haematological Malignancies.

4 Montserrat ARNAN, ${ }^{1}$ Carlota GUDIOL, ${ }^{2,3}$ Laura CALATAYUD, ${ }^{4,5}$ Josefina

5 LIÑARES, ${ }^{4,5}$ M. Ángeles DOMINGUEZ, ${ }^{4}$ Montserrat BATLLE, ${ }^{6}$ Josep $^{\mathbf{a}}$

6 RIBERA, ${ }^{6}$ Jordi CARRATALÀ,,${ }^{2,3}$ Francesc GUDIOL. ${ }^{2,3}$

7

8 Haematology Department, Hospital Duran i Reynals, Barcelona, ${ }^{1}$ Infectious Disease

9 Department, Hospital Universitari de Bellvitge, University of Barcelona, IDIBELL,

10 Barcelona, ${ }^{2}$ REIPI (Spanish Network for Research in Infectious Diseases), Instituto

11 de Salud Carlos III, Madrid, ${ }^{3}$ Microbiology Department, Hospital Universitari de

12 Bellvitge, University of Barcelona, IDIBELL, Barcelona, ${ }^{4}$ Ciber de Enfermedades

13 Respiratorias, ISCIII, Madrid, ${ }^{5}$ and Haematology Department, Hospital Germans

14 Trias i Pujol, Badalona, Barcelona, ${ }^{6}$ Spain.

16 Keywords: ESBL-Escherichia coli, neutropenia, faecal carriage, risk factors,

17 outcome

19 Corresponding author: Dr. Carlota Gudiol. Infectious Disease Department,

20 Hospital Universitari de Bellvitge, Feixa Llarga s/n, 08907 L'Hospitalet de Llobregat,

21 Barcelona, Spain. E-mail: cgudiol@iconcologia.net

22 Telephone: (+34) 932607625; Fax: (+34) 932607637

23 Montserrat Arnan: Hematology Department, Hospital Duran i Reynals, 1’Hospitalet 24 de Llobregat, Barcelona, Spain. 
25 Laura Calatayud: Microbiology Department, Hospital Universitari de Bellvitge,

26 1’Hospitalet de Llobregat, Barcelona, Spain.

27 Josefina Liñares: Microbiology Department, Hospital Universitari de Bellvitge, 28 1’Hospitalet de Llobregat, Barcelona, Spain.

29 M. Ángeles Domínguez: Microbiology Department, Hospital Universitari de 30 Bellvitge, 1’Hospitalet de Llobregat, Barcelona, Spain.

31 Montserrat Batlle: Hematology Department, Hospital Universitari Germans Trias i 32 Pujol, Badalona, Barcelona, Spain.

33 Josep $\mathbf{M}^{\mathbf{a}}$ Ribera: Hematology Department, Hospital Universitari Germans Trias i 34 Pujol, Badalona, Barcelona, Spain.

35 Jordi Carratalà: Infectious Disease Department, Hospital Universitari de Bellvitge, 36 l’Hospitalet de Llobregat, Barcelona, Spain.

37 Francesc Gudiol: Infectious Disease Department, Hospital Universitari de Bellvitge, 38 1’Hospitalet de Llobregat, Barcelona, Spain.

39 
47 Purpose: To assess the risk factors for, and the clinical relevance of faecal carriage by extended-spectrum $\beta$-lactamase producing Escherichia coli (ESBL-EC) in neutropenic cancer patients (NCP).

Methods: An observational prospective multicentre cohort study was conducted

51 during two years at two teaching hospitals. Patients with acute leukaemia or undergoing stem cell transplantation were included during neutropenia episodes. Rectal swabs were obtained at hospital admission and weekly thereafter until discharge or death. ESBL-EC colonized episodes were compared with non-colonized episodes. ESBL-EC strains were studied by PCR and isoelectric focusing, and molecular typing was performed by PFGE.

57 Results: Among 217 episodes of neutropenia, the prevalence of ESBL-EC faecal carriage was $29 \%, 14 \%$ at hospital admission. Multivariate analysis identified previous antibiotics as the only independent risk factor for ESBL-EC faecal colonization (OR: 5.38; 95\% CI: 2.79-10.39). Analysis of ESBL-EC isolates revealed

61 a polyclonal distribution with CTX-M predominance (81.3\%). E. coli bacteraemia 62 was mainly caused by non-ESBL producing strains and its rate was similar in both 63 groups (13\% vs 11\%). We found no association between ESBL-EC carriage and an 64 increased risk of ESBL-EC bacteremia or a negative influence on other clinical 65 outcomes, including length of hospitalisation, early and overall mortality rates.

66 Conclusions: ESBL-EC faecal colonization is frequent in NCP but difficult to 67 identify by epidemiological or clinical features on presentation. Prior antibiotic 68 therapy is the major associated risk factor. In this setting colonization does not appear 
69 to have a significant clinical relevance. Thus, routine testing for ESBL-EC faecal

70 carriage does not seem to be beneficial.

71

72

73

74

75

76

77

78

79

80

81

82

83

84

85 
87 Infectious complications remain a major cause of morbidity and mortality in

88 neutropenic cancer patients (NCP). Escherichia coli is one of the commonest causes

89 of bacteraemia in this patient population, and so the development of antimicrobial

90 resistance in E. coli is a cause of special concern in NCP. In the last decade, there has

91 been a marked increase in colonization and infection due to quinolone-resistant $E$.

92 coli, probably as a consequence of the widespread use of fluoroquinolone prophylaxis

93 for febrile neutropenic episodes [1-4]. More recently, other resistance mechanisms in

94 E. coli, such as extended-spectrum $\beta$-lactamase (ESBL) production, have been

95 described in a range of epidemiological scenarios worldwide [5-9]. Recently we

96 reported a high prevalence of ESBL-EC faecal colonization among neutropenic

97 patients with haematological malignancies [10]. However, no prospective studies

98 have investigated the associated risk factors and the clinical relevance of this carriage

99 in these patients.

101 METHODS

102 Setting, patients, and study design

103 An observational prospective multicentre cohort study was conducted between 1 May

1042006 and 31 December 2007 in Barcelona, Spain, at two teaching hospitals: Hospital

105 Duran i Reynals (hospital A) and Hospital Germans Trias i Pujol (hospital B).

106 The study population comprised consecutive adult patients with acute leukaemia or

107 undergoing haematopoietic stem cell transplantation, and who received chemotherapy

108 and developed grade IV neutropenia. The same patient could be included more than 
once for different neutropenia episodes, provided there was an interval of two months

110 in between and no history of previous colonization or infection by ESBL-E coli.

111 Information regarding baseline characteristics, clinical data, rectal swabs, empirical

112 antibiotic therapy and outcomes was carefully recorded in a computerized database.

113 Rectal swabs were obtained at hospital admission and weekly thereafter until

114 discharge or death. To assess risk factors for intestinal colonization by ESBL-EC, we

115 compared baseline and demographic characteristics of colonized and non-colonized

116 episodes. We also compared these two groups in order to assess the clinical relevance

117 of faecal colonization. Following our institutional guidelines, most febrile neutropenic

118 episodes were empirically treated with the combination of a broad-spectrum

119 cephalosporin or a carbapenem, plus an aminoglycoside for the first 48 hours. The 120 initial empirical antibiotic treatment was not modified according to the results of the 121 rectal swabs. No antibacterial prophylaxis was administered during the study period.

122 This study was approved by the ethics committee of our institution.

\section{Definitions}

125 Grade IV neutropenia was defined as an absolute neutrophil count $<500 / \mathrm{mm}^{3}$. Prior 126 antibiotic therapy was defined as the receipt of any systemic antibiotic within one 127 month before colonization, or one month before admission in non-colonized patients. 128 Early mortality was defined as death for any cause within 7 days of admission and 129 overall mortality as death by any cause during hospitalisation. 
132 Rectal swabs were cultured on three plates: MacConkey agar alone and supplemented 133 with cefotaxime $(2 \mu \mathrm{g} / \mathrm{ml})$ and ceftazidime $(4 \mu \mathrm{g} / \mathrm{ml})$ [11]. E. coli ATCC 25922 (non134 ESBL strain) and $K$. pneumoniae ATCC 700603 (ESBL-strains) were used as 135 controls. Identification of E. coli strains and their antibiotic susceptibility testing were 136 performed using commercial panels from Microscan® system (SIEMENS). 137 Susceptibility or resistance to antimicrobial agents was defined according to CLSI 138 criteria, and ESBL production screening was detected by double-disk synergy test 139 [12]. The genetic relatedness of E. coli strains was tested by pulsed field gel 140 electrophoresis (PFGE). The whole DNA was digested with XbaI. Strains that 141 differed in three o fewer bands were considered as belonging to the same cluster [13]. 142 ESBLs were characterized by a multiplex PCR [14]. One strain per neutropenia 143 episode was studied.

\section{Statistical analysis}

146 The prevalence of intestinal colonization by ESBL-EC was calculated as the 147 percentage of carriers among the total number of episodes included. Colonized and 148 non-colonized episodes were compared using univariate analysis with the chi-square 149 test for categorical variables and Student's t-test or the Mann-Whitney U-test, as 150 appropriate, for continuous variables, and multivariate logistic regression analysis was 151 performed to determine the independent risk factors related to intestinal colonization.

152 Multivariable conditional logistic-regression analysis of factors potentially associated 153 with ESBL-EC acquisition and mortality included all statistically significant variables 154 in univariate analysis, gender and age, and all clinically important variables, whether 
they were statistically significant or not [15]. The analysis was performed with the stepwise logistic-regression model of the SPSS software package (SPSS).

\section{RESULTS}

159 During the study period, 217 episodes of neutropenia (130 hospital A and 87 hospital

160 B) from 162 patients were studied (78 hospital A and 84 hospital B). The median 161 number of episodes per patient was $1.3(1-5)$. The mean number of stool samples 162 obtained from each group (colonized and non-colonized episodes) was similar (4.55 \pm 1631.73 vs. $4.62 \pm 1.91$ samples). Overall, ESBL-EC strains were isolated from faeces of $16463(29 \%)$ of the 217 episodes studied, $29(13 \%)$ of them on hospital admission. Table 1651 shows the main baseline and demographic characteristics of all episodes compared 166 by groups. No significant differences were found regarding the majority of 167 characteristics analysed. Univariate analysis identified prior antibiotics $(62 \%$ in the 168 colonized group vs. $25 \%$ in the non-colonized group; $p<0.001$ ) as the only factor 169 associated with ESBL-EC colonization. An unconditional logistic regression model 170 with ESBL-EC colonization as the dependent variable and adjusted for age, gender, 171 presence of central venous catheter (CVC) and previous antibiotic therapy, also 172 identified previous antibiotic therapy as an independent risk-factor (OR: 5.38; 95\% 173 CI: 2.79-10.39; $p<0.001)$ (Table 2).

174 Sixty of the 63 ESBL-EC strains were available for typing and ESBL 175 characterization. The ESBLS identified among the 60 available strains in order of 176 frequency were: CTX-M-9 group (55\%, 33/60), CTX-M-1 (26.7\%, 16/60), SHV $177(15 \%, 9 / 60)$, and TEM $(3.3 \%, 2 / 60)$. Four isolates carried simultaneously an ESBL 178 enzyme and an OXA type $\beta$-lactamase [(3 strains harboured simultaneously a CTX- 
M-1 ESBL and OXA, and 1 strain harboured a TEM ESBL and OXA). Fifty-three different PFGE patterns were found among the 60 neutropenia episodes. Five patients

181 had two different neutropenia episodes, and only two of them were colonized by an 182 ESBL-EC strain of the same cluster in the two episodes. The remaining three patients 183 with two different neutropenia episodes were colonized by ESBL-EC strains of 184 different PFGE patterns. The spread of three clones (EC1, EC2 and EC3) carrying 185 bla $a_{\mathrm{CTX}-\mathrm{M} 14} \mathrm{ESBL}$ was detected among six patients of hospital B: EC1 in four patients, 186 EC2 in two patients and EC3 in two patients (Figure 1). Resistance rates to non- $\beta$ 187 lactam antibiotics were as follows: quinolones 75\%, trimethoprim-sulfamethoxazole $18866.7 \%$, gentamicin 30\%, and tobramycin 33.3\%. All strains remained fully 189 susceptible to amikacin and carbapenems. The $16.7 \%$ of the strains were resistant to 190 amoxicillin-clavulanic and $3.4 \%$ to piperacillin-tazobactam. Table 3 shows the 191 antibiotic resistance rates, the resistance patterns, and PFGE patterns of the 60 192 available ESBL-EC strains, according to ESBL type.

193 Outcomes of the study population according to the ESBL-EC faecal carriage are 194 shown in Table 4. Out of the 217 episodes of neutropenia, 67 and 123 episodes of 195 infection were documented in group 1 and group 2 respectively, either clinically or 196 microbiologically, with no differences between groups. Among the microbiologically 197 documented infections, non-ESBL-EC strains accounted for 15 infections in group 1 198 and 15 infections in group 2, of which 8 and 13 were bacteraemias and 7 and 2 were 199 urinary tract infections respectively. ESBL-EC strains caused one infection 200 (bacteraemia) in group 1 and two infections (bacteraemia and urinary tract infection) 201 in group 2. One of the 2 patients with ESBL-EC bacteraemia received cefepime as the 202 initial empirical antibiotic therapy, which was switched to imipenem 48 hours later; 203 the patient died 4 days after the onset of bacteraemia due to cerebral haemorrhage. 
204 The second patient was empirically treated with imipenem from the beginning, 205 presenting a good clinical response. There were no statistical differences between 206 groups regarding other outcomes during admission, including early and overall 207 mortality.

\section{DISCUSSION}

210 In recent years, a significant increase in the incidence of ESBL-EC faecal carriage has

211 been detected in a variety of settings, including hospitalised patients, outpatients and 212 healthy individuals [16-20]. We recently reported a high prevalence of ESBL-EC 213 colonization in NCP [10]. This figure, reaching a rate of $31.8 \%$, was higher than that

214 found by other investigators in non-neutropenic populations [20-22]. It would be 215 interesting to know the corresponding data from other cancer centres.

216 Patients analysed in the present study can be considered representative of a 217 homogeneous population of adults with haematological malignancies and 218 neutropenia. There were no significant differences in their baseline characteristics or 219 clinical features, between patients who presented ESBL-EC faecal carriage and those 220 who did not. The only variable found to be an independent risk factor for ESBL-EC 221 colonization was previous antibiotic exposure, which has been widely recognized as a 222 risk factor for different ESBL-EC infections, especially bacteraemia and urinary tract 223 infections, by several authors in non-neutropenic populations $[5,7,9,23,24]$. The use of 224 prophylactic antibiotics, mostly quinolones, to prevent infection in febrile neutropenia 225 is a controversial issue in the literature [25]. Taking into account that antibiotic 226 exposure is a well defined risk factor for ESBL-EC colonization, it is reasonable to 227 speculate that if our patients had received routine antibiotic prophylaxis with 
quinolones for febrile neutropenic episodes, the subsequent ESBL-EC colonization rate would have been much higher. In our study, the presence of a CVC at admission was more frequently found in the colonised group. Nevertheless, we could not find a

231 reasonable explanation for this finding, thus, we considered it irrelevant.

232 Molecular typing and characterization of the ESBL-EC strains showed a high clonal 233 diversity among the isolates, with a clear predominance (81\%) of ESBL type CTX-M. 234 This finding is in good agreement with previous reports performed in the same 235 geographic area and also in several parts of the world [19,24,26]. Antibiotic resistance 236 by ESBL-EC strains to non $\beta$-lactam antibiotics was high, as previously reported $237[23,24]$.

238 As expected, infections caused by $E$. coli were frequent in our study, but we did not 239 find a correlation between the detection of ESBL-EC faecal colonization and the risk 240 of developing an ESBL-EC infection. In fact, the rate of non ESBL-EC bacteraemia 241 was around $10 \%$ in both groups, whereas the rate of ESBL-EC bacteraemia was less 242 than $2 \%$. This may indicate that, in spite of the presence of ESBL-EC colonies in the 243 selective plates, non ESBL-EC strains constitute the predominant E. coli population in 244 the faecal flora. Moreover, the occurrence of two episodes of ESBL-EC infection (one 245 episode of bacteraemia and one urinary tract infection) among the group of non246 colonised patients is remarkable. One possible explanation is the potential lack of 247 sensitivity of the screening test in the selective plates. Accordingly, our findings 248 suggest that testing faecal carriage for the presence of ESBL-EC strains may not be 249 cost effective.

250 On the other hand, as the number of persons colonized by ESBL-EC strains appears to 251 be expanding in the community, one would expect clinical infections caused by these 
strains to increase, albeit slowly. The therapeutic options for ESBL-EC infections are

253 limited, since these strains are considered to be resistant to all oxyiminobetalactams

254 and frequently present associated resistance to other antimicrobial families such as 255 aminoglycosides and quinolones [27]. This limitation is of special concern in NCP 256 and may lead to universal use of carbapenems as empirical therapy for the febrile 257 episodes. However, in view of our current knowledge, this practice should be 258 undertaken with caution and assessed in controlled trials.

259 Despite a number of strengths (prospective and multicentre study) our study has some 260 limitations that should be acknowledged. First, the study was conducted in a small 261 geographical area, which may not reflect the situation in different epidemiological 262 settings. And second, the small number of patients with ESBL-EC infections may 263 have not allowed us to find differences in outcomes between both groups.

264 In conclusion, in our study we found that ESBL-EC faecal colonization is frequent in 265 NCP but difficult to identify by epidemiological or clinical features on presentation. 266 Prior antibiotic therapy and is the major associated risk factor. Although E. coli 267 remains a frequent cause of infection in NCP, ESBL-EC bacteraemia is still rare. 268 Overall, the clinical relevance of this colonization appears to be low, and it does not 269 have a significant negative influence on the outcomes. In this setting, routine testing 270 for ESBL-EC faecal carriage does not seem to be beneficial. 
275 This study was supported by research grant REIPI RD06/0008 from the Ministerio de

276 Sanidad y Consumo, Instituto de Salud Carlos III, Spanish Network for the Research

277 in Infectious Diseases, and by research grant 051610 from Fundació la Marató de

278 TV3, Generalitat de Catalunya, Barcelona.

279

The funding sources had no role in the study design, the collection, analysis

280 and interpretation of the data or the decision to submit the manuscript for publication.

281 Only the authors had full access to the data files for the study. The authors do not

282 have any relationship that may constitute a dual or conflicting interest.

283

284

285

286

287

288

289

290

291

292

293

294

295

296

297 


\section{REFERENCES:}

299 1. Carratalà J, Fernández-Sevilla A, Tubau F et al (1995) Emergence of quinolone300 resistant Escherichia coli bacteremia in neutropenic patients with cancer who have 301 received prophylactic norfloxacin. Clin Infect Dis 20:557-60.

2. Carratalà J, Fernandez-Sevilla A, Tubau F et al (1996) Emergence of fluoroquionolne-resistant Escherichia coli in fecal flora of cancer patients receiving norfloxacin prohylaxis. Antimicrob Agents Chemother 40:503-05.

3. Yoo JH, Huh DH, Choi JH et al (1997) Molecular epidemiological analysis of quinolone-resistant Escherichia coli causing bacteremia in neutropenic patients

4. Zinner SH (1999) Changing epidemiology of infections in patients with neutropenia and cancer: emphasis on gram-positive and resistant bacteria. Clin Infect Dis 29: 490-4.

5. Ortega M, Marco F, Soriano A et al (2009) Analysis of 4758 Escherichia coli bacteremia episodes: predictive factors for isolation of an antibiotic-resistant strain and their impact on outcome. J Antimicrob Chemother 63:568-74.

6. Pitout JD, Laupland KB (2008) Extended-spectrum beta-lactamase-producing

317 7. Rodríguez-Baño J, Alcalá JC, Cisneros JM et al (2008) Community infections 318 caused by extended-spectrum $\beta$-lactamase-producing Escherichia coli. Arch $319 \quad$ Intern Med 168:1897-902.

320 8. Rodriguez-Baño J, Paterson DL (2006) A change in the epidemiology of 321 infections due to extended-spectrum beta-lactamase-producing organisms. Clin Infect Dis 42:935-7. 
9. Trecarichi EM, Tumbarello M, Spanu T et al (2009) Incidence and clinical impact of extended-spectrum- $\beta$-lactamase (ESBL) production and fluoroquinolone resistance in bloodstream infections caused by Escherichia coli in patients with hematological malignancies. J Infect 58:299-307.

10. Calatayud L, Arnan M, Liñares J et al (2008) Prospective study of fecal colonization by extended-spectrum $\beta$-lactamase-producing Escherichia coli in neutropenic patients with cancer. Antimicrob Agents Chemother 52:4187-90.

11. Peña C, Pujol M, Ardanuy C et al (1998) Epidemiology and successful control of a large outbreak due to Klebsiella pneumoniae producing extended-spectrum betalactamases. Antimicrob Agents Chemother 42:53-8.

12. Clinical and Laboratory Standards Institute (2007) Performance Standards for Antimicrobial Susceptibility Testing: Fifteenth Informational Supplement M100S15. CLSI, Wayne, PA, USA.

13. Tenover FC, Arbeit RD, Goering RV et al (1995) Interpreting chromosomal DNA restriction patterns produced by pulsed-field gel electrophoresis: criteria for bacterial strain typing. J Clin Microbiol 33:2233-9.

14. Fang H, Ataker F, Hedin G et al (2008) Molecular epidemiology of extendedspectrum beta-lactamases among Escherichia coli isolates collected in a Swedish hospital and its associated health care facilities from 2001 to 2006. J Clin

343 15. Hosmer DW, Lemeshow S (2000) Logistic regression: variable selection. In: 344 Hosmer DW, Lemeshow S, eds. Applied logistic regression. 2nd edn. New York: $345 \quad$ John Willey \& Sons, pp 92-115. 
16. Ben-Ami R, Schwaber MJ, Navon-Venezia S et al (2006) Influx of extendedspectrum $\beta$-lactamase-producing enterobacteriaceae into the hospital. Clin Infect Dis 42:925-34.

17. Castillo García FJ, Seral García C, Pardos de la Gandara M et al (2007) Prevalence of fecal carriage of ESBL-producing Enterobacteriaceae in hospitalized and ambulatory patients during two non-outbreak periods. Eur J Clin Microbiol Infect Dis 26:77-8.

18. Kader AA, Kumar A (2007) Fecal carriage of extended-spectrum $\beta$-lactamaseproducing Escherichia coli and Klebsiella penumoniae in patients and asymptomatic healthy individuals. Infect Control Hosp Epidemiol 28:1114-16.

19. Rodríguez-Baño J, López-Cerero L, Navarro MD et al (2008) Faecal carriage of extended-spectrum $\beta$-lactamase-producing Escherichia coli: prevalence, risk factors and molecular epidemiology. J Antimicrob Chemother 62:1142-49.

20. Valverde A, Coque TM, Sánchez-Moreno MP et al (2004) Dramatic increase in prevalence of fecal carriage of extended-spectrum $\beta$-lactamase-producing 361 Enterobacteriaceae during nonoutbreak situations in Spain. J Clin Microbiol 42:4769-75.

21. Mirelis B, Navarro F, Miró E et al (2003) Community transmission of extendedspectrum betalactamases. Emerg Infect Dis 9:1024-25.

22. Miró E, Mirelis B, Navarro F et al (2005) Surveillance of extended-spectrum betalactamases from clinical samples and faecal carriers in Barcelona, Spain. J Antimicrob Chemother 56:1152-55.

23. Peña C, Gudiol C, Tubau F et al (2006) Risk-factors for acquisition of extendedspectrum $\beta$-lactamase producing Escherichia coli among hospitalised patients. Clin Microbiol Infect 12:279-84. 
24. Ben-Ami R, Rodríguez-Baño J, Arslan H et al (2009) A multinational survey of risk factors for infection with extended-spectrum $\beta$-lactamase-producing Enterobacteriaceae in nonhospitalized patients. Clin Infect Dis 49:682-90.

25. Imran H, Tleyjeh Im, Arndt CA et al (2008) Fluoroquinolone prophylaxis in patients with neutropenia: a meta-analysis of randomized placebo-controlled trials. Eur J Clin Microbiol Infect Dis 27:53-63.

26. Rodríguez-Baño J, Navarro MD, Romero L et al (2006) Bacteremia due to extended-spectrum $\beta$-lactamase-producing Escherichia coli in the CTX-M era: A new clinical challenge. Clin Infect Dis 43:1407-14.

27. Paterson DL, Bonomo RA (2005) Extended-spectrum beta-lactamases: a clinical update. Clin Microbiol Rev 18:657-86. 
Table 1. Baseline characteristics of the study population according to the extendedspectrum $\beta$-lactamase $E$. coli faecal carriage.

\begin{tabular}{|c|c|c|c|c|}
\hline Characteristics & Total & $\begin{array}{c}\text { ESBL-EC } \\
\text { carriage }\end{array}$ & $\begin{array}{c}\text { ESBL-EC } \\
\text { non-carriage }\end{array}$ & $p$ \\
\hline No. of episodes & 217 & 63 & 154 & - \\
\hline $\operatorname{Sex}(M / F)$ & $132 / 85$ & $35 / 28$ & $97 / 57$ & 0.3 \\
\hline Mean age (yr, range) & $51.39 \pm 12.29$ & $50.95 \pm 11.92$ & $51.57 \pm 12.47$ & 0.7 \\
\hline \multicolumn{5}{|l|}{ Underlying disease } \\
\hline Acute leukaemia & $141(65 \%)$ & $36(57 \%)$ & $105(68 \%)$ & - \\
\hline Malignant Lymphoma & $31(14 \%)$ & $13(21 \%)$ & $18(12 \%)$ & - \\
\hline Multiple myeloma & $34(15 \%)$ & $11(17 \%)$ & $23(15 \%)$ & - \\
\hline Myelodysplastic Syndrome & $7(3 \%)$ & $3(5 \%)$ & $4(3 \%)$ & - \\
\hline Myeloproliferative disease & $4(3 \%)$ & 0 & $4(3 \%)$ & - \\
\hline Haematopoietic stem cell transplant & $99(46 \%)$ & $31(49 \%)$ & $68(44 \%)$ & 0.5 \\
\hline Previous chemotherapy (6 months) & $148(68 \%)$ & $42(67 \%)$ & $106(69 \%)$ & 0.7 \\
\hline Health-care contact & $172(79 \%)$ & $47(75 \%)$ & $125(81 \%)$ & 0.3 \\
\hline $\begin{array}{l}\text { Central venous catheter at } \\
\text { admission }\end{array}$ & $99(45 \%)$ & $22(35 \%)$ & $76(50 \%)$ & 0.048 \\
\hline Urinary catheter at admission & $5(2 \%)$ & $2(3 \%)$ & $3(2 \%)$ & 0.6 \\
\hline Previous antibiotic therapy & $77(36 \%)$ & $39(62 \%)$ & $38(25 \%)$ & $<0.001$ \\
\hline
\end{tabular}

398 NOTE: ESBL-EC: extended-spectrum $\beta$-lactamase $E$. coli 
400 Table 2. Independent risk factors for faecal extended-spectrum $\beta$-lactamase-producing 401 E. coli carriage.

\begin{tabular}{|l|c|c|}
\hline Risk-factor & Adjusted OR (95\% CI) & $p$ \\
\hline Age (years, mean) & $0.99(0.97-1.02)$ & 0.73 \\
\hline Gender (female) & $1.39(0.72-2.67)$ & 0.32 \\
\hline Central venous catheter & & 0.015 \\
\hline Previous antibiotic therapy & $0.43(0.22-0.85)$ & $<0.001$ \\
\hline
\end{tabular}

402

403

404

405

406

407

408

409

410

411

412 
413 Table 3. Antibiotic resistance, PFGE patterns and resistance patterns of 60 extended-

414 spectrum $\beta$-lactamase (ESBL) producing E. coli strains according to ESBL type.

415

\begin{tabular}{|c|c|c|c|c|}
\hline Antibiotic & $\begin{array}{c}\text { CTX-M9 } \\
(n=33)\end{array}$ & $\begin{array}{l}\text { CTX-M1 } \\
(n=16)\end{array}$ & $\begin{array}{l}\text { SHV } \\
(n=9)\end{array}$ & $\begin{array}{l}\text { TEM } \\
(\mathrm{n}=2)\end{array}$ \\
\hline & \multicolumn{4}{|c|}{ No of resistant strains } \\
\hline $\begin{array}{l}\text { Amoxicillin- } \\
\text { clavulanic acid }\end{array}$ & 2 & 4 & 3 & 1 \\
\hline $\begin{array}{l}\text { Piperacillin- } \\
\text { tazobactam }\end{array}$ & 0 & 0 & 2 & 0 \\
\hline Ciprofloxacin & 26 & 11 & 8 & 0 \\
\hline Gentamicin & 14 & 4 & 0 & 0 \\
\hline Tobramycin & 13 & 6 & 0 & 1 \\
\hline Amikacin & 0 & 0 & 0 & 0 \\
\hline Cotrimoxazole & 25 & 10 & 5 & 0 \\
\hline $\begin{array}{l}\mathrm{N}^{\circ} \text { different PFGE } \\
\text { patterns }\end{array}$ & 27 & 15 & 9 & 2 \\
\hline $\begin{array}{l}\text { Resistance patterns } \\
\left(\mathrm{N}^{\circ} \text { of strains }\right)\end{array}$ & $\begin{array}{l}\text { Cip, Gen, Tob, SxT (11) } \\
\text { Cip, SxT (11) } \\
\text { Cip (4) } \\
\text { SxT (3) } \\
\text { Cip, Gen, Tob (2) } \\
\text { Cip, Gen (1) } \\
\text { S (1) }\end{array}$ & $\begin{array}{l}\text { Cip, SxT (3) } \\
\text { Cip (3) } \\
\text { Cip, Gen, Tob, SxT (2) } \\
\text { Cip, Tob, SxT (2) } \\
\text { SxT (2) } \\
\text { Gen, Tob, SxT (1) } \\
\text { Cip, Gen, Tob (1) } \\
\text { S (2) }\end{array}$ & $\begin{array}{l}\text { Cip (4) } \\
\text { Cip, SxT (4) } \\
\text { SxT (1) }\end{array}$ & $\begin{array}{l}\text { Tob (1) } \\
\text { S (1) }\end{array}$ \\
\hline
\end{tabular}

416 NOTE: ESBL-EC: extended-spectrum $\beta$-lactamase $E$. coli 

Table 4. Outcomes of the study population according to the extended-spectrum $\beta$ -

419 lactamase faecal carriage.

\begin{tabular}{|c|c|c|c|}
\hline Characteristics & $\begin{array}{c}\text { ESBL-EC carriage } \\
\qquad \begin{array}{c}\mathrm{N}=63(\%)\end{array}\end{array}$ & $\begin{array}{c}\text { ESBL-EC non- } \\
\text { carriage } \\
\mathrm{N}=154(\%)\end{array}$ & $p$ \\
\hline One or more febrile episodes & $61(97)$ & $145(94)$ & 0.3 \\
\hline Infections per episode (mean \pm SD) & $1.57 \pm 0.99$ & $1.41 \pm 0.72$ & 0.3 \\
\hline Episodes of infection & 67 & 123 & - \\
\hline Clinically documented & $6(9)$ & $7(6)$ & 0.4 \\
\hline Microbiologically documented & $61(91)$ & $116(94)$ & 0.4 \\
\hline Non-ESBL-EC infections & $15(22)$ & $15(12)$ & 0.07 \\
\hline Bactaraemia & $8(13)$ & $13(11)$ & 0.7 \\
\hline Urinary tract infection & $7(10.4)$ & $3(2.4)$ & 0.018 \\
\hline ESBL-EC infections & $1(1.5)$ & $2(1.6)$ & 0.9 \\
\hline Bactaraemia & $1(1.5)$ & $1(1)$ & 0.7 \\
\hline Urinary tract infection & 0 & $1(1)$ & 0.5 \\
\hline Days of hospitalization (mean \pm SD) & $30.15 \pm 13.70$ & $29.05 \pm 12.11$ & 0.6 \\
\hline Days of antibiotic therapy (mean \pm SD) & $19.03 \pm 12.34$ & $19.39 \pm 13.55$ & 0.9 \\
\hline $\begin{array}{l}\text { Early mortality during hospitalization ( } 7 \\
\text { days) }\end{array}$ & $1(1.6)$ & 0 & 0.1 \\
\hline Overall mortality during hospitalization & $6(10)$ & $17(11)$ & 0.74 \\
\hline
\end{tabular}

420 NOTE: ESBL-EC: extended-spectrum $\beta$-lactamase $E$. coli 